\title{
Preparation and self-assembly behavior of polystyrene-block-poly (dimethylaminoethyl methacrylate) amphiphilic block copolymer using atom transfer radical polymerization
}

\author{
Y. J. Zhu' ${ }^{1}, Y$. B. $\operatorname{Tan}^{1,2 *}, X . D u^{1}$ \\ ${ }^{1}$ School of Chemistry and Chemical Engineering, Shandong University, Jinan 250100, People's Republic of China \\ ${ }^{2}$ Key Laboratory of Colloid and Interface Chemistry (Shandong University), Ministry of Education, Jinan 250100, \\ P. R. China
}

Received 15 November 2007; accepted in revised form 3 February 2008

\begin{abstract}
Asymmetric and semi-symmetric amphiphilic diblock copolymers polystyrene-block-poly (dimethylaminoethyl methacrylate) (PS- $b$-PDMAEMA) with the same PS block length of 62 repeat units and quite short (3 repeat units) or equivalent (47 repeat units) length of PDMAEMA have been prepared simply by varying the ratio of the bromine-terminated macroinitiator polystyrene (PS-Br) to DMAEMA using atom transfer radical polymerization (ATRP). The chemical structures and compositions of the PS- $b$-PDMAEMA block copolymers are studied by nuclear magnetic resonance (NMR) spectroscopy, gel permeation chromatography (GPC), and elementary analysis (EA). The self-assembly behaviors of copolymers in $\mathrm{N}, \mathrm{N}$-dimethyl formamide (DMF) with different $\mathrm{pH}$ and dioxane/water binary solvent mixture by direct dissolution method (DD), are studied by transmission electron microscopy (TEM), electron diffracting analysis (EDA), and energy-dispersive analysis of X-rays (EDAX) techniques. Transmission electron microscopy results suggest that asymmetric block copolymer $\mathrm{PS}_{62}-b$-PDMAEMA 3 (the numbers in the form of footnotes represent repeated units of each monomer in the copolymer) can form spherical core-shell micelles, large compound reverse micelles (LCRMs), hexagonal/rhombic phases, reverse hexagonal/rhombic phases, vesicles, reverse vesicles and necklace-like reverse micelles, controlled by common or selective solvent and $\mathrm{pH}$, while most of the aggregates of semi-symmetric $\mathrm{PS}_{62}-b$-PDMAEMA 47 are simply spherical, such as spherical core-shell micelles and reverse spherical core-shell micelles, besides hexagonal/rhombic phases. All above structures are controlled by three components of the free energy of aggregation: core-chain stretching, interfacial energy and intercoronal chain interaction.
\end{abstract}

Keywords: smart polymers, amphiphilic block copolymer, self-assembly, polymer synthesis

\section{Introduction}

Unlike the completely hydrophobic or hydrophilic block copolymers, amphiphilic block copolymer, especially containing $\mathrm{pH}$ and temperature-responsive polycationic poly (dimethylaminoethyl methacrylate) (PDMAEMA) as the hydrophilic segment, namely, polymeric surfactants, can provide many significant benefits to diverse applica- tions such as stabilizers, emulsifiers, or dispersants in industrial and pharmaceutical preparations [1-6]. They are also useful in colloid chemistry and self-assembly chemistry [7-9].

Amphiphilic block copolymers polystyrene-blockpoly (dimethylaminoethyl methacrylate) (PS- $b$ PDMAEMA) have been synthesized via living anionic polymerization [10,11]. Anionic polymer-

*Corresponding author, e-mail: ybtan@sdu.edu.cn

(C) BME-PT and GTE 
ization is an excellent and well-established method for controlling copolymer architecture and obtaining narrow molecular weight distributions. However, this method has drawbacks such as the necessary purity of the solvent and of the monomer, performance under high-vacuum and quite low reaction temperature. The recent development of living/controlled radical polymerizations such as Atom Transfer Radical Polymerization (ATRP) $[12,13]$, Reversible Addition Fragmentation chain Transfer polymerization (RAFT) [14] and Stable Free Radical Polymerization (SFRP) [4, 15], has opened a new route to synthesize functional and architectural block copolymers PS- $b$-PDMAEMA with well-defined structures. This paper reports the synthesis of two types of amphiphilic AB diblock copolymers of different lengths of PDMAEMA segment with narrow polydispersities by ATRP using well-defined bromine-terminated polystyrene (PS-Br) as macroinitiator, simply by varying the ratio of the macroinitiator to DMAEMA: one is asymmetric (95 mol\% of PS) diblock copolymer $\mathrm{PS}_{62}-b$-PDMAEMA 3 (the numbers in the form of footnotes represent repeated units of each monomer in the copolymer) (Block 1), and the other is semisymmetric (57 mol\% of PS) $\mathrm{PS}_{62}-b$-PDMAEMA 47 (Block 2).

Amphiphilic block copolymer can not only be prepared by versatile synthetic approaches but also has multiple morphologies generated by its different chemical interactions between two blocks or environment. A vast array of structures by asymmetric amphiphilic block copolymers in solution has been identified and a detailed investigation of the thermodynamic and kinetic parameters that induce morphologies has been begun by Eisenberg's group [16-39]. Two methods for aggregate preparation, the water addition method (WA) and the direct dissolution method (DD), have been exploited for the mixed solvent system, which have important influences on aggregation morphology and size. For WA, the polymer is in a molecularly disperse solution of good solvent for both blocks initially, and the aggregation is induced by precipitant addition for one block at a low concentration. At high precipitant concentration, chain exchange is very slow, and thus, despite the same final binary solvent mixture composition, the thermodynamic aggregation is appreciably controlled at low precipitant concentration. For DD, polymer is added as solid to a binary solvent mixture, and swelling followed by fission can lead to more complex morphologies that may better reflect thermodynamic equilibrium [40]. Spherical micelles assembled in aqueous solutions formed by amphiphilic $\mathrm{PS}_{131}-b$-PDMAEMA 36 asymmetric diblock [41] and $\mathrm{PS}_{10-}-b$-PDMAEMA 2 P- $^{-}$ $b$-PS 10 triblock copolymers [42] by WA method have been studied by TEM. However, few works involve the self-assembly aggregations of PS- $b$ PDMAEMA controlled by polymer chain length and environment. In this paper, the correlation of several novel morphologies of linear diblock copolymers PS- $b$-PDMAEMA with different PDMAEMA block lengths in a binary solvent mixture (dioxane/water) using DD method and in pure organic solvents (DMF) with different $\mathrm{pH}$ are addressed. Multiple polymeric aggregation conformations are obtained by asymmetric $\mathrm{PS}_{62}-b$ $\mathrm{PDMAEMA}_{3}$ compared with semi-symmetric $\mathrm{PS}_{62}-b$-PDMAEMA 47 and their changing tendencies are discussed in detail.

\section{Experimental section}

\subsection{Materials}

Styrene (S, Aldrich) and dimethylaminoethyl methacrylate (DMAEMA, AR) were purified by reduced pressure distillation to remove inhibitor. The monomers were stored at $-5^{\circ} \mathrm{C}$ for later use. Benzyl bromide (AR) was normally distilled and stored under an argon atmosphere at $-5^{\circ} \mathrm{C}$. $\mathrm{CuBr}$ and 2,2'-bipyridyl were used as received without further purification.

\subsection{Synthesis of Macroinitiator}

In a typical polymerization experiment, $0.20 \mathrm{ml}$ ( $0.0017 \mathrm{~mol})$ benzyl bromide, $0.265 \mathrm{~g}(0.0017 \mathrm{~mol})$ 2,2'-bipyridyl and $0.244 \mathrm{~g}(0.0017 \mathrm{~mol}) \mathrm{CuBr}$ were placed in a dried $100 \mathrm{ml}$ three-necked flask which was flushed with nitrogen. Pre-degassed styrene $(22 \mathrm{ml}, 0.1923 \mathrm{~mol})$ was added to the flask immersed in an oil bath at $90^{\circ} \mathrm{C}$, and then the solution was magnetically stirred for $5 \mathrm{~h}$ under a nitrogen atmosphere. Over this period the originally red translucent polymeric solution turned dark and opaque. After the polymerization was completed, the polymer was diluted by $20 \mathrm{ml}$ THF, and then precipitated in excess methanol after passing through an alumina column. The white powder was 
purified by re-dissolution in THF and reprecipitation in methanol, and then dried at $60^{\circ} \mathrm{C}$ under vacuum.

\subsection{Synthesis of block copolymerization}

Two shares of mixtures containing $1.161 \mathrm{~g}$ PS-Br macroinitiator, $0.019 \mathrm{~g}(0.133 \mathrm{mmol}) \mathrm{CuBr}$, and $0.021 \mathrm{~g}$ (0.133 mmol) 2,2'-bipyridyl, $27 \mathrm{ml} \mathrm{DMF}$ were respectively introduced into two $50 \mathrm{ml}$ glass vials under nitrogen atmosphere. The ampoules were immersed in an oil bath at $90^{\circ} \mathrm{C}$ and the ATRPs were started by adding $0.432 \mathrm{ml}$ $(2.556 \mathrm{mmol})$ and $4.321 \mathrm{ml} \quad(25.562 \mathrm{mmol})$ DMAEMA respectively. The reactions were left for $24 \mathrm{~h}$ with continuous stirring. After the polymerizations were completed, the former block copolymer 1 (Block 1) was precipitated in methanol while the later (Block 2) in ion-free water after passing through an alumina column, and dried at $60^{\circ} \mathrm{C}$ under vacuum.

\subsection{Sample preparation}

The samples were prepared by dissolved in pure DMF and dioxane/water (v/v: 94/6) binary solvent mixture using DD method with the same polymer concentration $(1 \mathrm{wt} \%)$. The $\mathrm{pH}$ of copolymer solutions in DMF was regulated by addition amount of $p$-toluene sulphonic acid.

\subsection{Characterization}

Nuclear Magnetic Resonance Spectra (NMR). The ${ }^{1} \mathrm{H}$ NMR spectra were registered by using an AVANCE-400MHz (Bruker) spectrophotometer. $\mathrm{CDCl}_{3}$ sample solutions (5-10 wt\%) were analyzed in tubs of $5 \mathrm{~mm}$, using TMS as internal standard.

Gel Permeation Chromatography (GPC). The analyses were carried out by using a Waters 515 chromatograph equipped with 2 columns Waters Styragel HT 3 and Waters Styragel HT 4, and a dif- ferential refractive index detector (Waters 2410). The temperature was maintained at $35^{\circ} \mathrm{C}$ in a Waters column oven. A mixed solvent of $\mathrm{Et}_{3} \mathrm{~N} /$ THF (v/v: 1/99) (HPLC grade) was used as eluent at $1 \mathrm{ml} / \mathrm{min}$ flow rate, and the samples were prepared at concentration of about $5-10 \mathrm{mg} / \mathrm{ml}$ in $\mathrm{Et}_{3} \mathrm{~N} / \mathrm{THF}$. A standard curve was built up by using monodisperse polystyrene samples of molecular weight comprised between 1360 and $2320000 \mathrm{~g} / \mathrm{mol}$ using Et 3 N/THF as solvent.

Elementary Analysis (EA) Analysis of $\mathrm{C}, \mathrm{H}$, and $\mathrm{N}$ elements was done using a Vario EL III (ELEMENTAR) element analyzer manufactured by elementary analysis system, Germany.

Transmission Electron Microscopy (TEM). The inner structures of the aggregations are observed by Hitachi-800 transmission electron microscope (Hitachi Co., Tokyo, Japan) operating at $150 \mathrm{kV}$ equipped with Philips PV9900 energy-dispersive analysis of X-rays (EDAX) apparatus and D/max$\mathrm{rB}$ electron diffracting (ED) apparatus. A drop of solution is dispersed onto a solidified epoxy-coated electron microscope grid, stained with 1 wt $\%$ potassium phosphotungstate aqueous solution as the negative staining agent, and then quenched with liquid nitrogen.

\section{Results and discussion}

\subsection{Synthesis and characterization}

ATRP is an excellent and well-established method for obtaining block copolymers using simple experimental protocols and a large variety of the available monomers, especially for preparation of amphiphilic block copolymers with well-defined architecture and narrow molecular weight distribution [43-50]. This example demonstrates, in the best way, the features that must be considered for a successful employment of ATRP in block copolymer synthesis. The facile process of polymerization of the amphiphilic block copolymer is depicted in Figure 1.

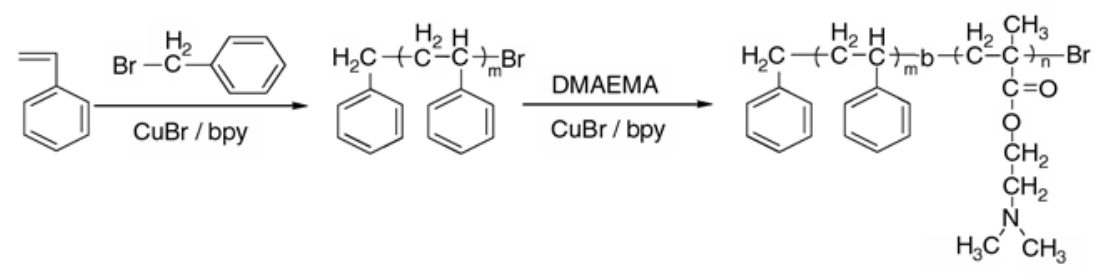

Figure 1. Synthesis of PS- $b$-PDMAEMA copolymer 


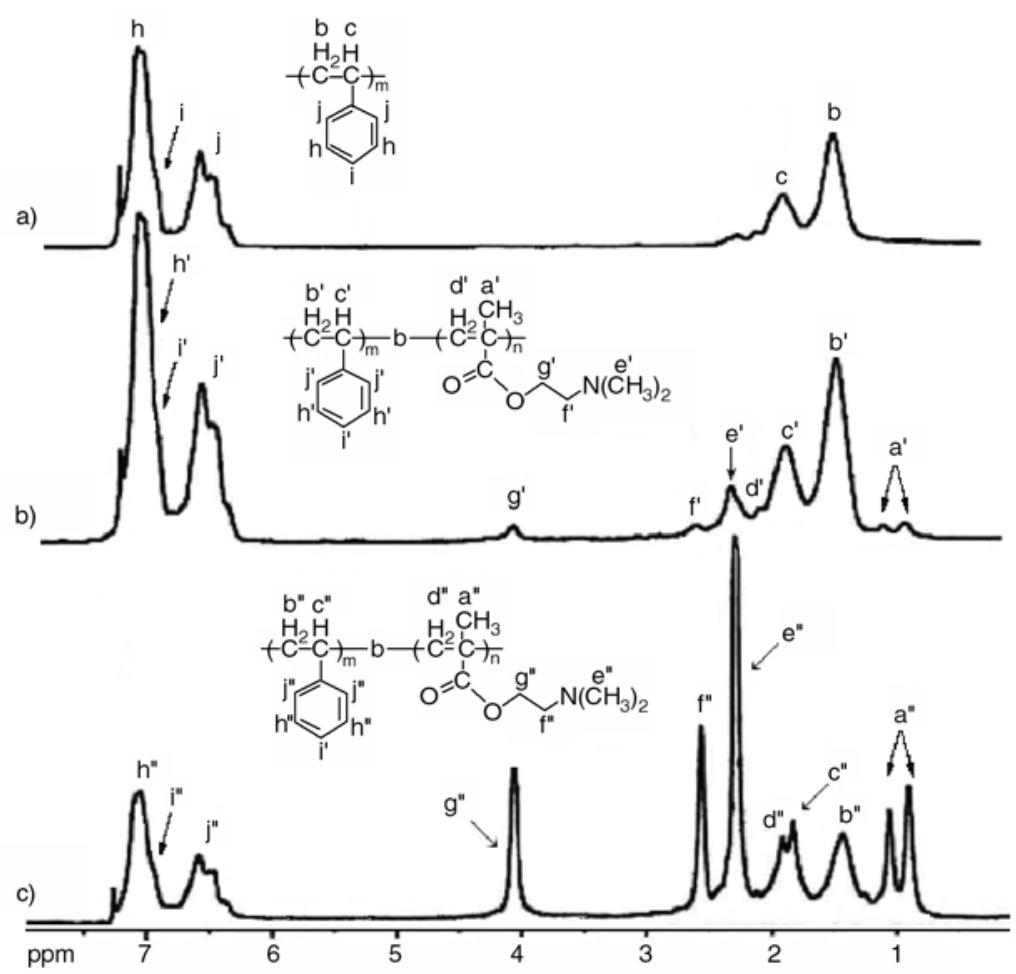

Figure 2. ${ }^{1} \mathrm{H}$ NMR spectra of the macroinitiator and the block copolymer sample in different forms: (a) Macroinitiator PS-Br; (b) Block 1; (c) Block 2

Figure 2 shows typical ${ }^{1} \mathrm{H}$ NMR spectra of bromine-terminated polystyrene as macroinitiator and the block copolymer. Two scales of diblock copolymers, with the same PS block but with different degrees of polymerization of DMAEMA were synthesized. Signals assigned to PS moiety (at $\delta=1.4-1.8 \mathrm{ppm}$ and $\delta=6.4-7.1 \mathrm{ppm}$ ) and the PDMAEMA moiety (at $\delta=0.8-1.1 \mathrm{ppm}, \delta=$ $2.2-2.6 \mathrm{ppm}$, and $\delta=4.1-4.2 \mathrm{ppm}$ ) are clearly visible.

Single, and symmetrical molecular weight distribution peaks were obtained for all samples (Figure 3). The molecular weight of copolymers mainly depended on the molar ratio of DMAEMA monomer to macroinitiator of PS-Br. The $M_{w} / M_{n}$ values of the polymers measured by gel permeation chromatography (GPC) were 1.20-1.26. The clear shift of the GPC traces toward the higher molecular weights indicated that the macromolecular chains were extended with the second monomer (DMAEMA) in a well-controlled way to form a block copolymer PS- $b$-PDMAEMA. For the sake of clearness, we took the GPC trace of Block 2 for example, which had the longest interval between PS-Br and block copolymer peaks. The GPC trace of Block 2 with longer PDMAEMA segment was a

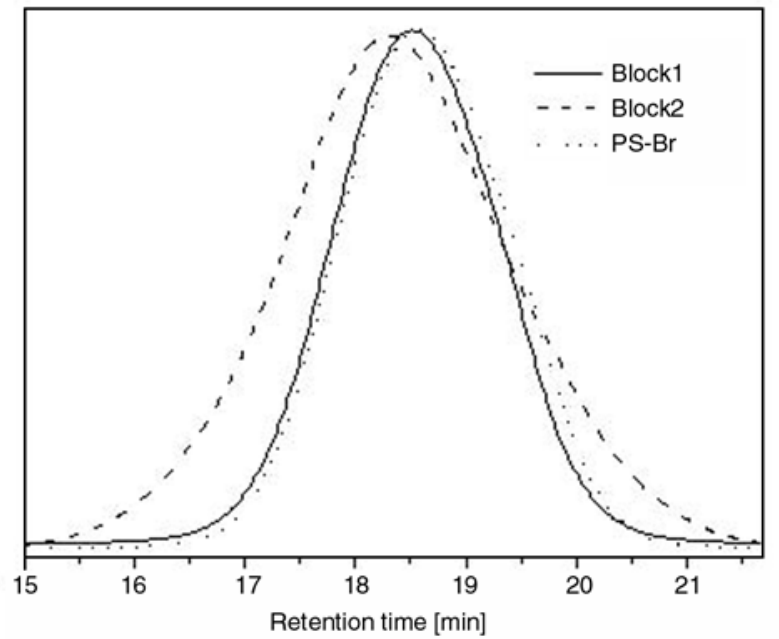

Figure 3. GPC traces of samples obtained in the synthesis of PS- $b$-PDMAEMA (their structures and the final characteristics shown in Table 1) using $\mathrm{PS}-\mathrm{Br}$ as macroinitiator via ATRP process

monodisperse and narrow distributed single peak without shoulder peak, which was evidence that all PS macromolecules were bromine-terminated and had taken part in the polymerization reactions as macroinitiator, that was no PS homopolymer was present in block copolymers. The presence of PDMAEMA in PS- $b$-PDMAEMA was also impossible while the precipitants (methanol for Block 1 
Table 1. Molecular characteristic of the macroinitiator and the copolymers PS- $b$-PDMAEMA determined by GPC, ${ }^{1} \mathrm{H}$ NMR and EA analyses

\begin{tabular}{|c|c|c|c|c|c|c|c|c|c|}
\hline \multirow{2}{*}{ Sample } & \multirow{2}{*}{$\begin{array}{l}\mathbf{M}_{n, \mathbf{t h}^{\mathbf{a}}} \\
{[\mathrm{g} / \mathrm{mol}]}\end{array}$} & \multirow{2}{*}{$\begin{array}{c}\text { Conversion } \\
{[\%]}\end{array}$} & \multirow{2}{*}{$\begin{array}{c}\mathbf{M}_{\mathbf{n}}{ }^{\mathbf{b}} \\
{[\mathrm{g} / \mathrm{mol}]}\end{array}$} & \multirow{2}{*}{$\begin{array}{c}\mathbf{M}_{w}{ }^{b} \\
{\left[g / m_{0 l}\right]}\end{array}$} & \multirow{2}{*}{$\mathbf{M}_{\mathbf{w}} / \mathbf{M}_{\mathbf{n}} \mathbf{b}$} & \multirow{2}{*}{ Polymer structureb } & \multicolumn{3}{|c|}{ m(St)/n(DMAEMA) } \\
\hline & & & & & & & H NMR & EA & GPC \\
\hline Macroinitiator & 6599 & 54.56 & 6429 & 7829 & 1.218 & $\mathrm{PS}_{62}-\mathrm{Br}$ & - & - & - \\
\hline Block 1 & 8184 & 69.42 & 6857 & 8196 & 1.195 & $\mathrm{PS}_{62}-b$-PDMAEMA 3 & 34.2 & 36.5 & 22.7 \\
\hline Block 2 & 16569 & 43.65 & 13876 & 17525 & 1.263 & $\mathrm{PS}_{62}-b$-PDMAEMA 47 & 1.31 & 1.35 & 1.32 \\
\hline
\end{tabular}

aTheoretical number average molecular weight $\left(M_{n, t h}\right)$ calculated from the feed capacity. $M_{n, t h}=\left(M_{m} \times \mathrm{Conversion} \times[\mathrm{M}] /[\mathrm{I}]\right)+M_{i}$, where $M_{m}$ and $M_{i}$ are the molar masses of monomers and initiator (or macroinitiator), respectively, [M] and [I] are the concentration of monomer and initiator (or macroinitiator), respectively

bNumber average molecular weight $\left(M_{n}\right)$, weight average molecular weight $\left(M_{w}\right)$, polydispersity index $\left(M_{w} / M_{n}\right)$ and the segment length of the polymers, by GPC

and water for Block 2) were solvents for PDMAEMA homopolymer. Table 1 summarizes the conversions, compositions, theoretical and experimental molecular weights, and molecular weight distributions of a series of PS- $b$ PDMAEMA copolymers via atom transfer radical polymerization.

As the results of Nuclear Magnetic Resonance Spectra ( ${ }^{1} \mathrm{H}$ NMR), Gel Permeation Chromatography (GPC), and Elementary Analysis (EA), we have successfully synthesized a series of amphiphilic polymers with narrow molecular weight distributions.

\subsection{Self-assembly}

The conformational state of the polymers in solution, which is responsible for the compatibility phenomena, depends on the solubility parameter $(\delta)$. Dissolution of polymers is optimal where the $\delta$ values for polymer and solvent are similar (see Table 2) [51]. High dielectric constant $(\varepsilon)$ values and hydrogen bonding interaction improve the solubility of the corona block [52]. All aspects of the free energy of the aggregation are influenced by solvent. In a polar solvent, the hydrophobic part of the block copolymer will form the core of such micelles, whereas the hydrophilic block is expected to extend into the polar phase [53].
Here we report on the TEM study of the structural nature of PS- $b$-PDMAEMA in DMF with various protonated degrees and in dioxane/water binary solvent mixture (shown in Figure 4, 5, 6 and 9). Table 3 shows the $\mathrm{pH}$ - and solvent-dependence of the morphology and size measured from TEM images.

It was found that, with increasing $\mathrm{pH}$, the sequence of copolymer structures in solution follows the order of sphere and hexagonal phase mixtures, spheres, vesicles, pearl necklaces, and finally hexagonal phases. The vesicular morphology is produced by just changes in the solution $\mathrm{pH}$, which suggests that the morphological changes are induced by decreased repulsion (both steric and electrostatic) among the hydrophilic segments as a result of the protonation of PDMAEMA (by $p$-toluene sulphonic acid). All above structures have a phase separated core and a soluble corona controlled by three components of the free energy of aggregation: core-chain stretching, interfacial energy and intercoronal chain interaction $[36,54]$. The shade of color of the resulting aggregations can be controlled by adjusting the dyeing time in the negative staining agent.

The plan view electron micrograph images showed that the aggregations consisted of the hexagonal phase, rhombic phase and spherical micellar aggre-

Table 2. Solubilities of copolymers and homopolymers in various solvents

\begin{tabular}{|c|c|c|c|c|c|c|c|}
\hline \multirow{2}{*}{ Material } & \multirow{2}{*}{ H-bond ${ }^{b}$} & \multirow{2}{*}{$\begin{array}{c}\delta \\
{\left[\left(\mathrm{cal}^{\prime} \mathrm{cm}^{3}\right)^{1 / 2}\right]}\end{array}$} & \multirow{2}{*}{$\varepsilon^{c}$} & \multirow{2}{*}{ PS } & \multirow{2}{*}{ PDMAEMA } & \multicolumn{2}{|c|}{ PS- $b$-PDMAEMA ${ }^{d}$} \\
\hline & & & & & & 95\% (Block 1) & $57 \%$ (Block 2) \\
\hline DMF & $\mathrm{m}$ & 12.1 & 36.7 & $++^{\mathrm{a}}$ & + & ++ & ++ \\
\hline Water & $\mathrm{s}$ & 23.4 & 80.1 & $--^{\mathrm{a}}$ & ++ & -- & -- \\
\hline 1,4-Dioxane & $\mathrm{m}$ & 10.0 & $2.21^{*}$ & ++ & -- & ++ & $+t_{a}$ \\
\hline
\end{tabular}

$\mathrm{a}(++)$ readily soluble $(10 \%, \mathrm{r} . \mathrm{t}) ;(--)$ insoluble; $(+)$ soluble.

bH-bond: hydrogen bond; s-strongly m-moderately ib.

cdielectric constant $\left(20^{\circ} \mathrm{C}\right) ;{ }^{*} 25^{\circ} \mathrm{C}$; from reference 53 .

${ }^{d}$ weight fractions of styrene in the copolymers are $95 \%$ and $57 \%$, respectively. 

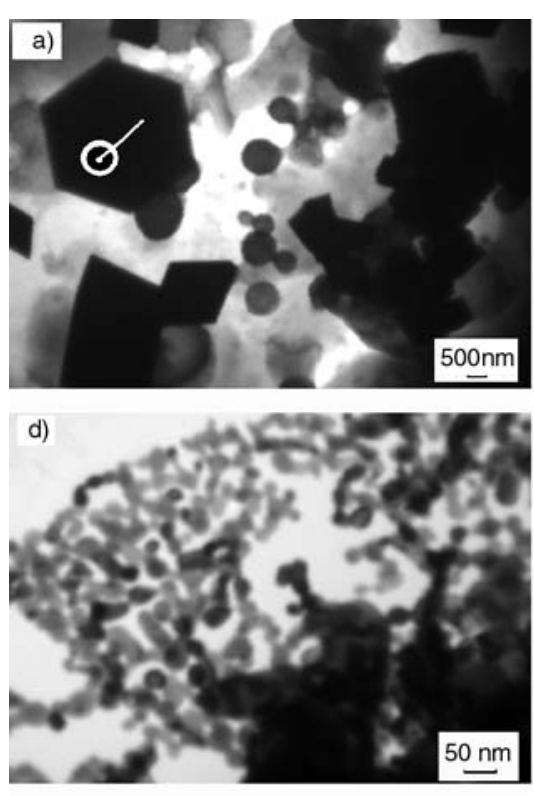
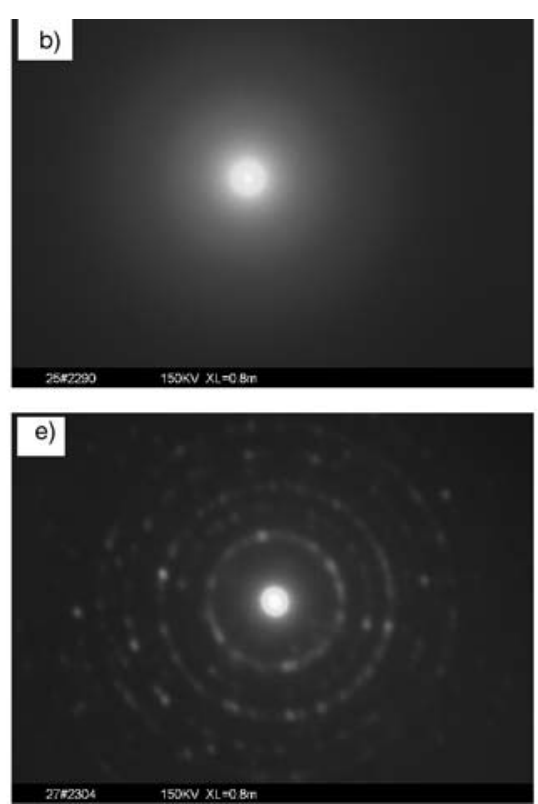

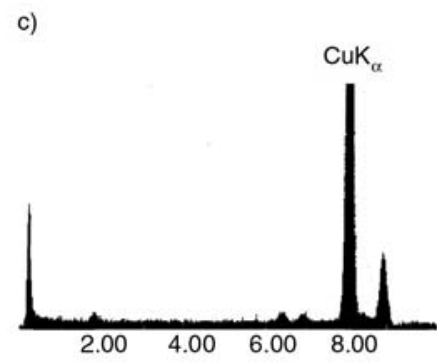

f)

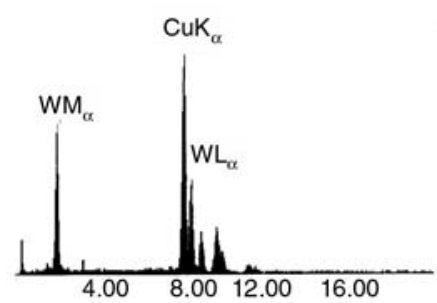

Figure 4. TEM images, electron diffracting (ED) patterns, and energy-dispersive analysis of X-rays (EDAX) spectra of the aggregations self-assembled by 1 wt $\% \mathrm{PS}_{62}-b$-PDMAEMA 3 in DMF at $\mathrm{pH}=1(\mathrm{a}, \mathrm{b}, \mathrm{c})$ and crystal of negative staining agent $(\mathrm{d}, \mathrm{e}, \mathrm{f})$ used in TEM

Table 3. Summary of the effect of solvent polarity or $\mathrm{pH}$ on the morphology for PS- $b$-PDMAEMA

\begin{tabular}{|c|l|c|l|c|}
\hline \multicolumn{2}{|c|}{ Solvent } & \multicolumn{2}{|c|}{ Block 1 } & \multicolumn{2}{c|}{ Block 2 } \\
\hline & \multicolumn{1}{|c|}{ Morphology } & Size $^{\mathbf{a}}[\mathbf{n m}]$ & \multicolumn{1}{c|}{ Morphology } & Size $^{\mathbf{a}}[\mathbf{n m}]$ \\
\hline $\mathrm{DMF} \quad \mathrm{pH}=1$ & hexagonal/rhombic phases & $443-1131$ & hexagonal/rhombic phases & $714-988$ \\
\hline & spherical micelles & $668-1310$ & & \\
\hline $\mathrm{pH}=2$ & spherical micelles & $420-768$ & hexagonal/rhombic phases & $226-1710$ \\
\hline $\mathrm{pH}=4$ & vesicles & $370-690$ & spherical micelles & $7-9$ \\
\hline $\mathrm{pH}=5$ & necklace-like micelles & $208-320$ & spherical micelles & $4-5$ \\
\hline & hexagonal/rhombic phases & $1948-3638$ & & $73-194$ \\
\hline dioxane/water & large compound micelles & $89-499$ & spherical micelles & \\
\hline & vesicles & $48-232$ & & \\
\hline
\end{tabular}

aThe values listed in the table are the diameters of micelles and vesicles or the side lengths of hexagonal/rhombic particles.

gations self-assembled by $1 \mathrm{wt} \% \quad \mathrm{PS}_{62}-b-$ $\mathrm{PDMAEMA}_{3}$ in DMF at $\mathrm{pH}=1$ and crystal of negative staining agent, as shown in Figure $4 \mathrm{a}$ and Figure 4d. To our knowledge, only inorganic compounds and a few organic compounds can form highly symmetrical crystal. It seems incredible that an amorphous polymer, such as PS- $b$-PDMAEMA, can self-assemble into a highly symmetrical structure such as a hexagonal phase or a rhombic phase. To confirm the composition of the hexagonal particles, the corresponding selected area energy-dispersive analysis of X-rays (EDAX) spectrum is shown in Figure 4c, compared with the EDAX spectrum of potassium phosphotungstate in Figure 4f, by which elemental composition of aggregations were analyzed to shed light on the structure. The strong $\mathrm{Cu}$ signal is caused by the sample holder (copper grid). We did not detect any signals corresponding to inorganic compounds, indicating our purification of the powder of the copolymer of PS- $b$ PDMAEMA eliminated any inorganic impurity. The selected area diffraction patterns are shown in Figure $4 \mathrm{~b}$ and Figure 4e. The intensity of the rings from the hexagonal phases is hazy and decrescent indicating that the phases do not have a preferred orientation just as crystal. Contrarily, the superimposed rings originating from electron diffraction from crystal of potassium phosphotungstate were composed of bright spots. We judge that this lack of preferred orientation in hexagonal phase might be due to the undefined arrangement of macromolecules holding long chains and a great deal of atoms. By combining these three techniques, detailed information on the elemental component and the two-dimensional structure of aggregations could be determined. 

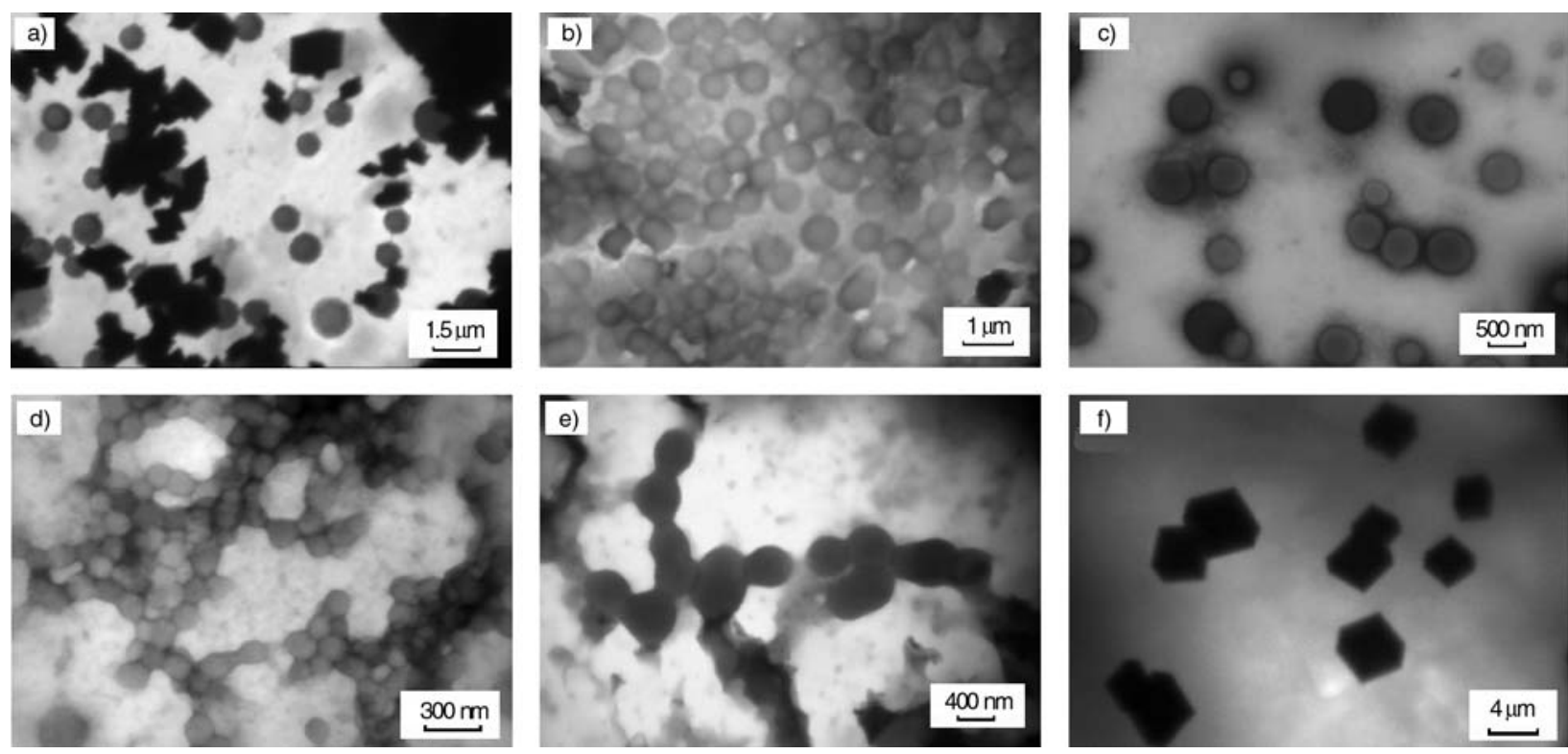

Figure 5. TEM images of the aggregations self-assembled by $1 \mathrm{wt} \% \mathrm{PS}_{62}-b$-PDMAEMA 3 in DMF at $\mathrm{pH}=1$ (a); $\mathrm{pH}=2$ (b); $\mathrm{pH}=4$ (c) and $\mathrm{pH}=5(\mathrm{~d}, \mathrm{e}, \mathrm{f})$

First time Shen and Eisenberg reported of the formation of hexagonal particles self-assembled by $10.0 \mathrm{wt} \%$ asymmetric amphiphilic $\mathrm{PS}_{49}-b-\mathrm{PAA}_{7.2}$ in dioxane/water (w/w: 63/37) mixture [29]. They presumed that the inverted morphologies were so compact that no internal structure could be seen, probably because of the very small diameters of the hollow rod regions that the shot PAA blocks produced in the PS domain. Here we obtained much larger and regular hexagonal and rhombic structures in DMF self-assembled by not only the asymmetric amphiphilic $\mathrm{PS}_{62}-b$-PDMAEMA 3 at $\mathrm{pH}=1$ (Figure 4a and 5a) and $\mathrm{pH}=5$ (Figure $5 \mathrm{f}$ ) but also the semi-symmetric $\mathrm{PS}_{62}-b$-PDMAEMA47 at $\mathrm{pH}=1$ (Figure 9a) and $\mathrm{pH}=2$ (Figure 9b). For the coexistence of large dimensioned hexagonal/ rhombic structure and spherical micelles, we believe that the hexagonal/rhombic structure is formed by the second aggregation of very small PS- $b$ PDMAEMA micelles with higher total surface areas and energy than the larger micelles (Figure 7a and 10a).
For linear PS- $b$-PDMAEMA in polar solvent, the corona repulsion force and the core dimension determined the morphology. In DMF solvent, the corona repulsion force of PDMAEMA blocks with high degree of protonation controlled by addition amount of $p$-toluene sulphonic acid was dominant and thus the sphere morphology was observed. We can clearly see coexistence of spherical nanoparticles with the sizes between 700 and $1000 \mathrm{~nm}$, having a phase-separated PS core and a PDMAEMA corona (Figure $7 \mathrm{~b}$ and $10 \mathrm{~b}$ ), and hexagonal particles in Figure 5a. The hexagonal particles totally disappear and only spherical micelles exist when $\mathrm{pH}$ is further increased to 2 . The micelles have a nearly spherical shape and an average diameter of $500 \mathrm{~nm}$, as shown in Figure 5b.

When the $\mathrm{pH}$ of solution is increased, the size of morphologies, as listed in Table 3, decreases. Much smaller micelles with average diameters decreasing from about 8 to $5 \mathrm{~nm}$ are also formed by $\mathrm{PS}_{62}-b$ PDMAEMA $_{47}$ while the solution $\mathrm{pH}$ increases from 4 to 5 (Figure 9c and 9d). While longer corona
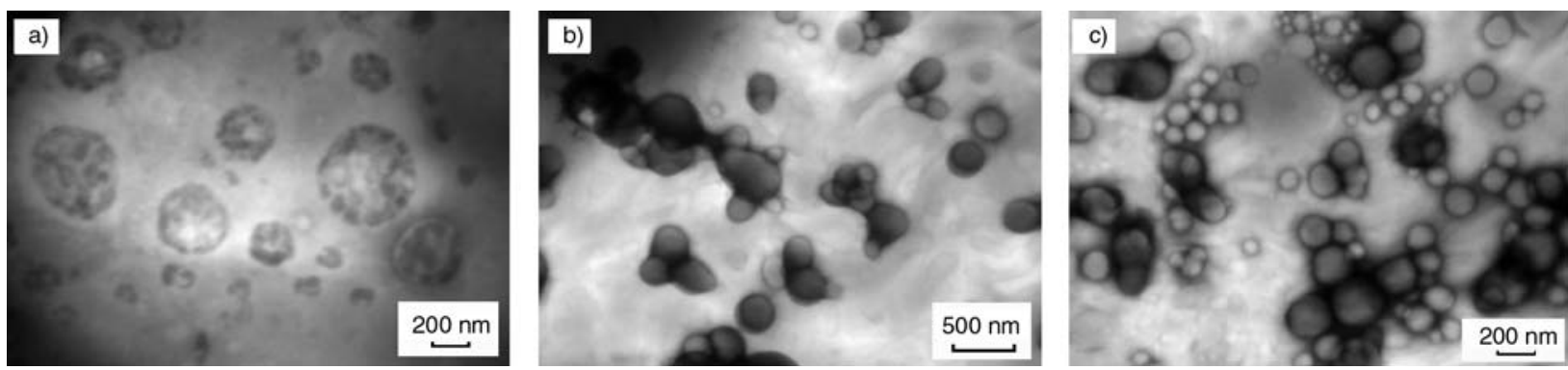

Figure 6. TEM images of the aggregations self-assembled by $1 \mathrm{wt} \% \mathrm{PS}_{62}-b-\mathrm{PDMAEMA}_{3}$ in dioxane/water (v/v: 94/6) 

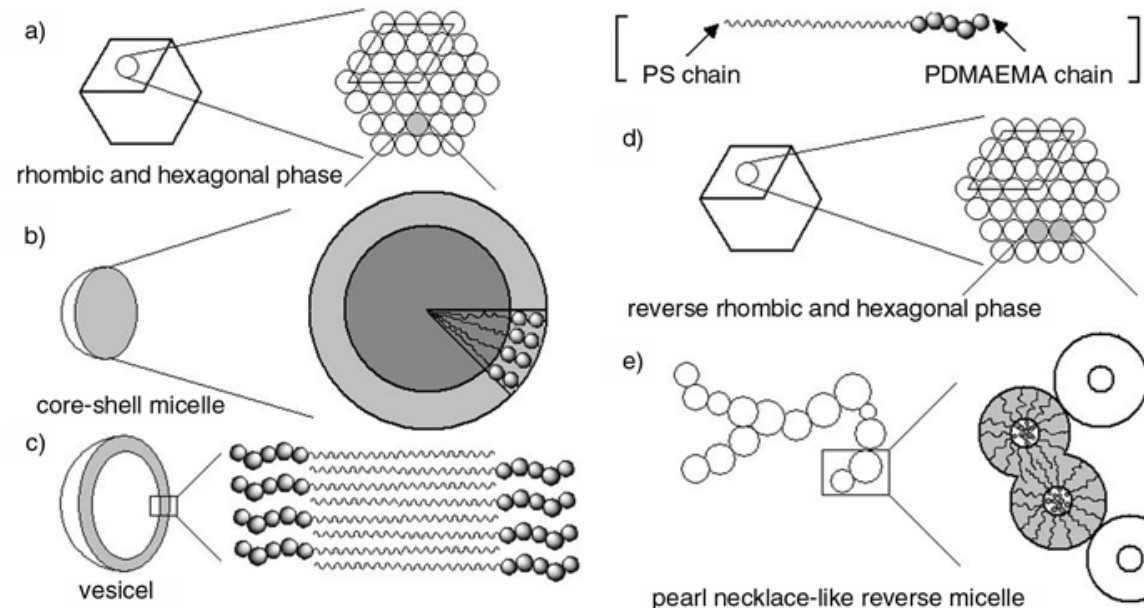

d)

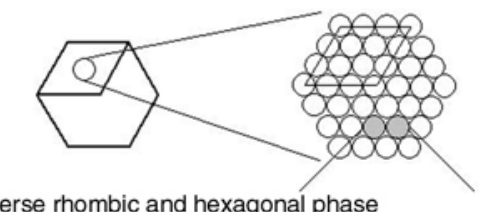

e)

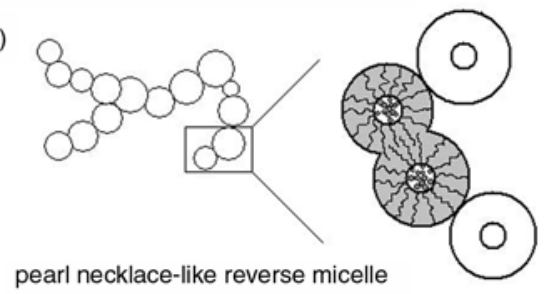

Figure 7. Proposed chain architectures of block copolymers PS $_{62}-b$-PDMAEMA 3 in the hexagonal/rhombic phase (a), spherical core-shell micelle (b), vesicle (c), reverse rhombic/hexagonal phase (d), and pearl necklace-like reverse micelle (e)

chain makes the micellar aggregation number decrease [30], the micelles formed by $\mathrm{PS}_{62}-b$ PDMAEMA $_{47}$ are smaller than those self-assembled by $\mathrm{PS}_{62}-b$-PDMAEMA 3 , though the former molecule is larger. For the weaker electrostatic repulsive force between corona PDMAEMA chains, smaller micelles are formed by $\mathrm{PS}_{62}-b$ PDMAEMA $_{47}$ in solution $\mathrm{pH}=5$ self-assembled by less molecules than $\mathrm{pH}=4$. Meanwhile, the quantity of spherical micelles also increases obviously.

Figure $5 c$ shows TEM image of vesicular aggregations self-assembled by $1 \mathrm{wt} \% \mathrm{PS}_{62}-b$-PDMAEMA 3 in $\mathrm{DMF}$ at $\mathrm{pH}=4$. Well defined closed bilayer structures are seen in the image. The contrast in the images Figure 5b and Figure $5 c$ can be directly understood as the vesicular structure with a dark halo surrounding the grayer interior. For $\mathrm{PS}_{62}-b$ $\mathrm{PDMAEMA}_{3}$, the weaker the electrostatic repulsive force is, the lower the morphological curvature is. Lower curvature makes the vesicular structure appear (Figure 7c).

Core-shell micelles are present again and coexist with sheer hexagonal phases self-assembled by
$1 \mathrm{wt} \% \mathrm{PS}_{62}-b$-PDMAEMA 3 in DMF at $\mathrm{pH}=5$. Their low magnification images are shown in Figure $5 \mathrm{~d}, 5 \mathrm{e}$ and Figure $5 \mathrm{f}$. The micelles are seen to have sizes down to $250 \mathrm{~nm}$ compared with micelles with a bigger average diameter of $500 \mathrm{~nm}$ in Figure $5 \mathrm{~b}$. Typically, with increasing $\mathrm{pH}$ condition, the micelles are connected by bridging chains yielding a bunch structure that exhibits interesting necklace shape, with occasional multifunctional beads maintaining the gelled network. The pearl necklace morphology is most likely a result of the acid driving the spheres to form rods. However, the glassy nature of the PS cores of the beads makes it impossible to complete the transformation. The pearl necklace thus represents an intermediate step between spheres and rods [54]. Furthermore, hexagonal and rhombic phases with side length on the order of $\sim 3 \mu \mathrm{m}$ are prepared in DMF at $\mathrm{pH}=5$ (Figure 5f). Their three-dimensional structures are especially important to be understood in the study of such large regions of the detailed image contrast in such polygonal shape.

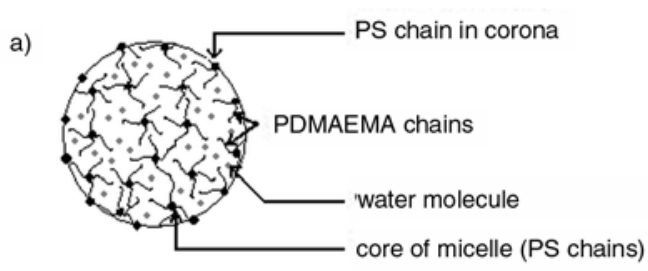

large compound reverse micelle (LCRM)

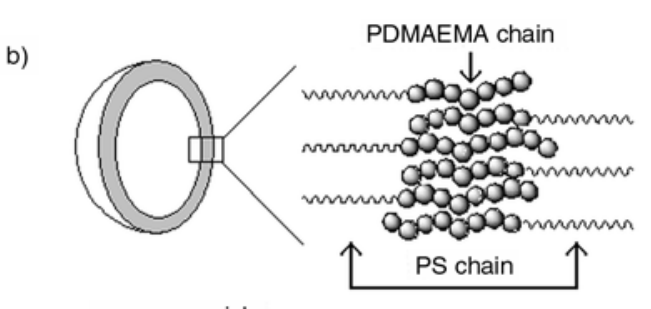

reverse vesicle

Figure 8. Proposed chain architectures of block copolymers $\mathrm{PS}_{62}-b-\mathrm{PDMAEMA}_{3}$ in the large compound reverse micelle (LCRM) (a), and reverse vesicle (b) 

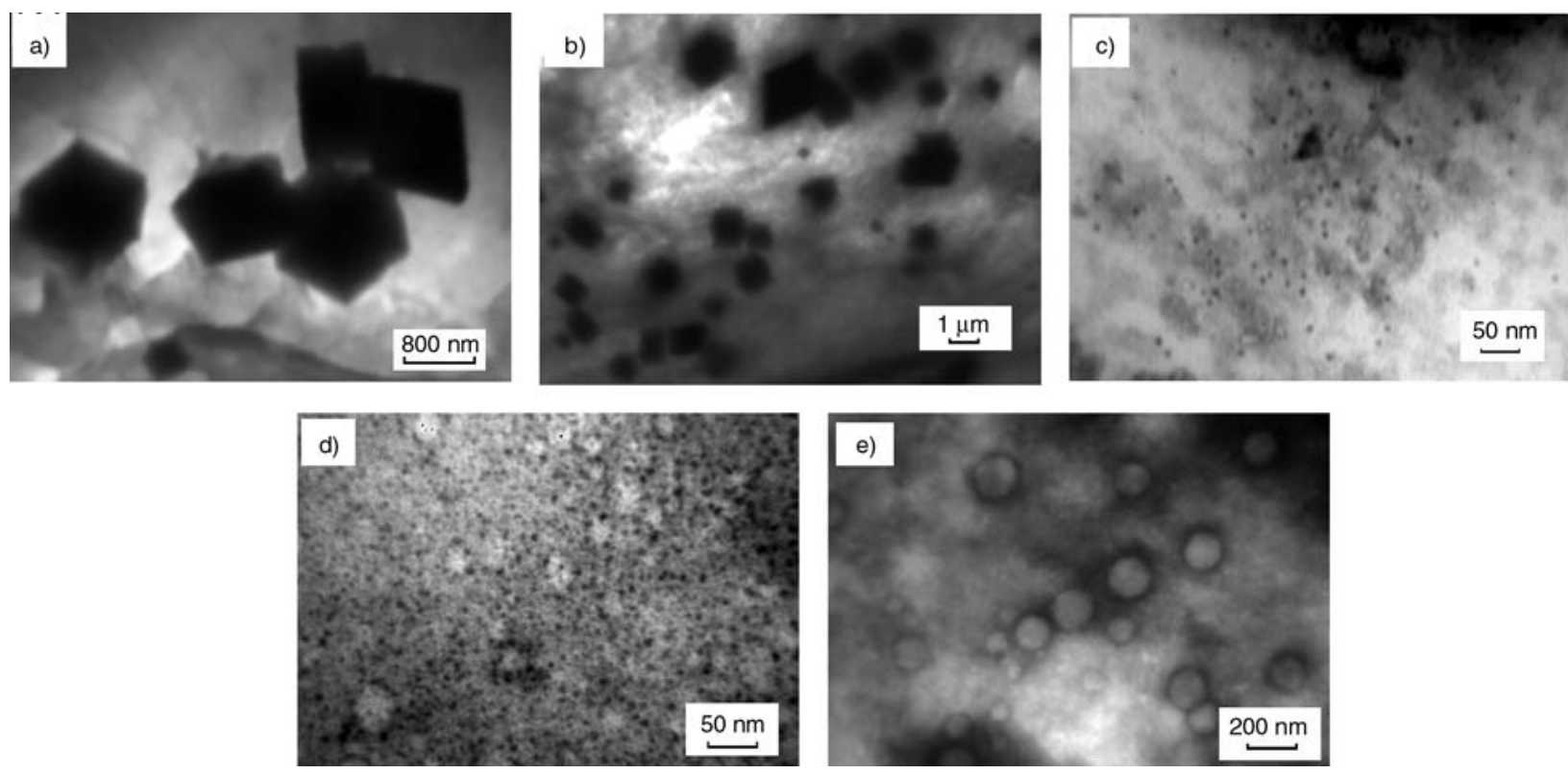

Figure 9. TEM images of the aggregates self-assembled by 1 wt $\% \mathrm{PS}_{62}-b$-PDMAEMA 47 in DMF at $\mathrm{pH}=1(\mathrm{a}) ; \mathrm{pH}=2$ (b); $\mathrm{pH}=4$ (c); $\mathrm{pH}=5$ (d); and in dioxane/water (v/v: 94/6) (e)

Because the protonation degree of PDMAEMA block in $\mathrm{PS}_{62}-b$-PDMAEMA 3 is much lower in $\mathrm{DMF}$ at $\mathrm{pH}=5$ than at $\mathrm{pH}=2$, the electrostatic repulsion should not contribute much to the smaller spherical morphology through vesicular morphology transition. The effect of the other two parameters, the interface tension at the core-corona interface and the stretching (deformation) of the core-forming blocks in the core, may affect the final morphology. As DMF is the common solvent for both PS and PDMAEMA blocks (Table 2), the polarity of PDMAEMA block decreases according to the $\mathrm{pH}$ increasing, which induces the interface tension at the core-corona interface decrease. It's likely that the PS stretching and decreased interface tension cause the morphology change form vesicle to pearl necklace-like reverse micelle with PS corona and PDMAEMA core. The fusion between

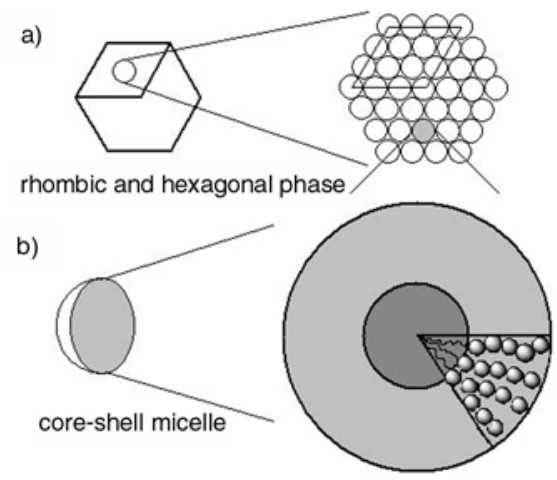

reverse micelles might be caused by interpenetration of PS blocks in corona (Figure 7e). We proposed that the hexagonal/rhombic structure is also a reverse phase and formed by the second aggregation of very small $\mathrm{PS}_{62}-b$-PDMAEMA 3 reverse micelles (Figure 7d).

With increasing hydrophobic characteristic of the solvent in dioxane/water (v/v: 94/6) mixture compared with DMF, we observed the large compound reverse micelles (LCRMs) which are spherical and highly polydisperse formed by secondary aggregation of reverse micelles self-assembled by $\mathrm{PS}_{62}-b$ $\mathrm{PDMAEMA}_{3}$ (Figure 6a). The LCRMs formation is illustrated that islands of PS chains are presumed in a continuous phase of core-forming polymer (PDMAEMA) all of which is surrounded by the solvent soluble PS corona (Figure 8a). The model is enlightened by Eisenberg who proposed the LCRMs
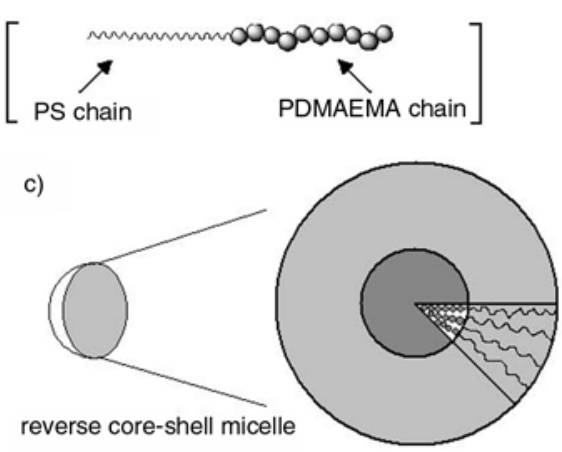

Figure 10. Proposed chain architectures of block copolymers $\mathrm{PS}_{62}-b$-PDMAEMA 47 in the hexagonal/rhombic phase (a), spherical core-shell micelle (b), and reverse spherical core-shell micelle (c) 
formed by PS- $b$-PAA in aqueous solution bore a strong resemblance to bulk reverse micelles, which present a further solution to the problem of how to arrange a large, insoluble block where the solubilizing block is very short [55]. Vesicles are also coexisting in the solvent mixture (Figure $6 \mathrm{~b}$ and $6 \mathrm{c}$ ).

A striking model of the proposed chain architectures of block copolymers $\mathrm{PS}_{63}-b$-PDMAEMA 3 in the hexagonal/rhombic phase, spherical core-shell micelle, vesicle, reverse hexagonal/rhombic phase, pearl necklace-like reverse micelle is proposed in Figure 7 and large compound reverse micelle (LCRM), reverse vesicle in Figure 8. For polystyrene with PDMAEMA hydrophilic end-caps and a molecular weight greater than small molecular surfactant, $\mathrm{PS}_{62}-b$-PDMAEMA 3 copolymers selfassemble into big size core-shell micelles or large compound reverse micelles (LCRMs) consisting of a hydrophilic core that is surrounded by a corona of PS segment. Typically, the higher $\mathrm{pH}$ condition of solvent decreases the electrostatic repulsion of these positively charged PDMAEMA head-groups due to the lower charge density, which causing the size of the aggregation decrease.

It is found that the aggregation configuration selfassembled by $1 \mathrm{wt} \% \mathrm{PS}_{62}-b$-PDMAEMA 47 (coreshell micelle) remains unchanged with $\mathrm{pH}$ shift in DMF from 4 to 5 . And also, in dioxane/water (v/v: 94/6) solvent mixture, only reverse core-shell micelles are obtained (Figure 9e). From the TEM results in Figure 9, the self-assembly microstructure of $\mathrm{PS}_{62}-b$-PDMAEMA 47 based on the aggregation mechanism as depicted in Figure 10 is proposed.

\section{Conclusions}

In this study, amphiphilic linear block copolymer with one block of polystyrene (PS) and another environmental-sensitive block of poly (dimethylaminoethyl methacrylate) (PDMAEMA) were successfully synthesized by atom transfer radical polymerization.

We report on transmission electron microscopy (TEM) based structural studies of the self-assembly behavior of PS- $b$-PDMAEMA. Indeed our experiments have reported changes in the structure of aggregations formed by $\mathrm{PS}_{62}-b$-PDMAEMA 3 resulting from changing in solvent and in charge density by adjusting solvent acidity. A rich variety in the image contrast is observed. At the same time,
$\mathrm{PS}_{62}-b$-PDMAEMA 47 copolymers are in the form of micelles in different solvent. Besides simple, core-shell micelles, large compound reverse micelles (LCRMs), vesicles, reverse vesicles, hexagonal phase, reverse hexagonal phase and pearl necklace-like micellar aggregates have been prepared from asymmetric diblock copolymers $\mathrm{PS}_{62}-b$-PDMAEMA 3 in dilute solution (1 wt $\%$ ). This study illustrates the chain architectures of block copolymers in the morphologies of molecular self-assembled nanostructures. Some of the aggregates may have potential applications in areas such as separations and drug delivery systems. When the PS block length is nearly equivalent to the PDMAEMA block length, most of the aggregates of semi-symmetric diblock copolymers $\mathrm{PS}_{62}-b$ PDMAEMA $_{47}$ in organic solvent (DMF) or binary solvent mixture (dioxane/water, v/v: 94/6) are spherical, with a core-shell structure.

We note that the larger size of aggregations is due to their electrostatic repulsion of these positively charged PDMAEMA head-groups and their low surface curvature associated energy. The participation of polymers in aggregations enhances the configuration stability caused by the viscosity between polymeric long chains. Moreover, their large surface-to-volume ratio makes them sensitive to environmental conditions, which are intimately linked to their structural reorganization, including size, shape, bulk (interior), and surface bonding. All above structures are controlled by three components of the free energy of aggregation: core-chain stretching, interfacial energy and intercoronal chain interaction.

\section{Acknowledgements}

The work was financially supported by National Natural Science Foundation of China (No. 20674045), Shandong Provincial Natural Science Foundation (No.Y2007B34) and the Scientific Research Foundation for the Returned Overseas Chinese Scholars, State Education Ministry.

\section{References}

[1] Alvarez-Lorenzo C., Barreiro-Iglesias R., Concheiro A., Iourtchenko L., Alakhov V., Bromberg L., Temchenko M., Deshmukh S., Hatton T. A.: Biophysical characterization of complexation of DNA with block copolymers of poly(2-dimethylaminoethyl) methacrylate, poly(ethylene oxide), and poly(propylene oxide). Langmuir, 21, 5142-5148 (2005). 
[2] Even M., Haddleton D. M., Kukulj D.: Synthesis and characterization of amphiphilic triblock polymers by copper mediated living radical polymerization. European Polymer Journal, 39, 633-639 (2003).

[3] Jiang X., Lok M. C., Hennink W. E.: Degradablebrushed PHEMA-PDMAEMA synthesized via ATRP and click chemistry for gene delivery. Bioconjugate Chemistry, 18, 2077-2084 (2007).

[4] Khelfallah N. S., Peretolchin M., Klapper M., Müllen $\mathrm{K}$.: Controlled radical polymerization of $\mathrm{N}, \mathrm{N}-$ dimethylaminoethyl methacrylate using triazolinyl as counter radical. Polymer Bulletin, 53, 295-304 (2005).

[5] Rakhmatullina E., Braun T., Kaufmann T., Spillmann H., Malinova V., Meier W.: Functionalization of gold and silicon surfaces by copolymer brushes using surface-initiated ATRP(a). Macromolecular Chemistry and Physics, 208, 1283-1293 (2007).

[6] Wang X., Winnik M. A., Manners I.: Synthesis and self-assembly of poly(ferrocenyldimethylsilane- $b$ dimethylaminoethyl methacrylate): Toward water-soluble cylinders with an organometallic core. Macromolecules, 38, 1928-1935 (2005).

[7] Baines F. L., Armes S. P., Billingham N. C., Tuzar Z.: Micellization of poly(2-(dimethylamino)ethyl methacrylate-block-methyl methacrylate) copolymers in aqueous solution. Macromolecules, 29, 8151-8159 (1996).

[8] Baines F. L., Billingham N. C., Armes S. P.: Synthesis and solution properties of water-soluble hydrophilichydrophobic block copolymers. Macromolecules, 29, 3416-3420 (1996).

[9] Baines F. L., Dionisio S., Billingham N. C., Armes S. P.: Use of block copolymer stabilizers for the dispersion polymerization of styrene in alcoholic media. Macromolecules, 29, 3096-3102 (1996).

[10] Costa A. C., Geoghegan M., Vlcek P., Composto R. J.: Block copolymer adsorption from a homopolymer melt to silicon oxide: Effects of nonadsorbing block length and anchoring block-substrate interaction. Macromolecules, 36, 9897-9904 (2003).

[11] Ni P., Zhang M., Ma L., Fu S.: Poly(dimethylamino) ethyl methacrylate for use as a surfactant in the miniemulsion polymerization of styrene. Langmuir, 22, 6016-6023 (2006).

[12] Zhang X., Matyjaszewski K.: Synthesis of welldefined amphiphilic block copolymers with 2(dimethylamino)ethyl methacrylate by controlled radical polymerization. Macromolecules, 32, 17631766 (1999).

[13] Zhao B., Brittain W. J.: Synthesis, characterization, and properties of tethered polystyrene- $b$-polyacrylate brushes on flat silicate substrates. Macromolecules, 33, 8813-8820 (2000).

[14] Xiong Q., Ni P., Zhang F., Yu Z.: Synthesis and characterization of 2-(dimethylamino)ethyl methacrylate homopolymers via aqueous RAFT polymerization and their application in miniemulsion polymerization. Polymer Bulletin, 53, 1-8 (2004).
[15] Lokaj J., Vlcek P., Kriz J.: Synthesis of polystyrenepoly(2-(dimethylamino)ethyl methacrylate) blockcopolymers by stable free-radical polymerization. Macromolecules, 30, 7644-7646 (1997).

[16] Allen C., Han J. N., Yu Y. S., Maysinger D., Eisenberg A.: Polycaprolactone- $b$-poly(ethylene oxide) copolymer micelles as a delivery vehicle for dihydrotestosterone. Journal of Controlled Release, 63, 275-286 (2000).

[17] Allen C., Maysinger D., Eisenberg A.: Nano-engineering block copolymer aggregates for drug delivery. Colloids and Surfaces B: Biointerfaces, 16, 3-27 (1999).

[18] Allen C., Yu Y., Maysinger D., Eisenberg A.: Polycaprolactone-b-poly(ethylene oxide) block copolymer micelles as a novel drug delivery vehicle for neurotrophic agents FK506 and L-685,818. Bioconjugate Chemistry, 9, 564-572 (1998).

[19] Bronich T. K., Kabanov A. V., Kabanov V. A., Yu K., Eisenberg A.: Soluble complexes from poly(ethylene oxide)-block-polymethacrylate anions and $\mathrm{N}$-alkylpyridinium cations. Macromolecules, 30, 3519-3525 (1997).

[20] Bronich T. K., Popov A. M., Eisenberg A., Kabanov V. A., Kabanov A. V.: Effects of block length and structure of surfactant on self-assembly and solution behavior of block ionomer complexes. Langmuir, 16, 481-489 (2000).

[21] Discher D. E., Eisenberg A.: Polymer vesicles. Science, 297, 967-973 (2002).

[22] Kabanov A. V., Bronich T. K., Kabanov V. A., Yu K., Eisenberg A.: Spontaneous formation of vesicles from complexes of block ionomers and surfactants. Journal of the American Chemical Society, 120, 9941-9942 (1998).

[23] Eisenberg A., Liu F. T.: Preparation and pH triggered inversion of vesicles from poly(acrylic acid)-blockpolystyrene-block-poly(4-vinyl pyridine). Journal of the American Chemical Society, 125, 15059-15064 (2003).

[24] Luo L. B., Eisenberg A.: Thermodynamic size control of block copolymer vesicles in solution. Langmuir, 17, 6804-6811 (2001).

[25] Luo L. B., Eisenberg A.: Thermodynamic stabilization mechanism of block copolymer vesicles. Journal of the American Chemical Society, 123, 1012-1013 (2001).

[26] Moffitt M., Vali H., Eisenberg A.: Spherical assemblies of semiconductor nanoparticles in water-soluble block copolymer aggregates. Chemistry of Materials, 10, 1021-1028 (1998).

[27] Savic R., Luo L., Eisenberg A., Maysinger D.: Micellar nanocontainers distribute to defined cytoplasmic organelles. Science, 300, 615-618 (2003).

[28] Shen H. W., Eisenberg A.: Morphological phase diagram for a ternary system of block copolymer PS(310)- $b$-PAA(52)/dioxane/ $\mathrm{H}_{2} \mathrm{O}$. Journal of Physical Chemistry, B, 103, 9473-9487 (1999). 
[29] Shen H. W., Eisenberg A.: Block length dependence of morphological phase diagrams of the ternary system of PS- $b$-PAA/dioxane/ $\mathrm{H}_{2} \mathrm{O}$. Macromolecules, 33, 2561-2572 (2000).

[30] Shen H. W., Zhang L. F., Eisenberg A.: Multiple pHinduced morphological changes in aggregates of polystyrene-block-poly(4-vinylpyridine) in $\mathrm{DMF} / \mathrm{H}_{2} \mathrm{O}$ mixtures. Journal of the American Chemical Society, 121, 2728-2740 (1999).

[31] Soo P. L., Eisenberg A.: Preparation of block copolymer vesicles in solution. Journal of Polymer Science, Part B: Polymer Physics, 42, 923-938 (2004).

[32] Yu G. E., Eisenberg A.: Multiple morphologies formed from an amphiphilic ABC triblock copolymer in solution. Macromolecules, 31, 5546-5549 (1998).

[33] Yu K., Eisenberg A.: Bilayer morphologies of selfassembled crew-cut aggregates of amphiphilic PS- $b$ PEO diblock copolymers in solution. Macromolecules, 31, 3509-3518 (1998).

[34] Yu Y., Eisenberg A.: Control of morphology through polymer-solvent interactions in crew-cut aggregates of amphiphilic block copolymers. Journal of the American Chemical Society, 119, 8383-8384 (1997).

[35] Yu Y., Zhang L., Eisenberg A.: Morphogenic effect of solvent on crew-cut aggregates of apmphiphilic diblock copolymers. Macromolecules, 31, 1144-1154 (1998).

[36] Zhang L., Eisenberg A.: Morphogenic effect of added ions on crew-cut aggregates of polystyrene- $b$-poly (acrylic acid) block copolymers in solutions. Macromolecules, 29, 8805-8815 (1996).

[37] Zhang L., Eisenberg A.: Formation of crew-cut aggregates of various morphologies from amphiphilic block copolymers in solution. Polymers for Advanced Technologies, 9, 677-699 (1998).

[38] Zhang L. F., Eisenberg A.: Thermodynamic vs kinetic aspects in the formation and morphological transitions of crew-cut aggregates produced by self-assembly of polystyrene- $b$-poly(acrylic acid) block copolymers in dilute solution. Macromolecules, 32, 2239-2249 (1999).

[39] Zhang L. F., Shen H. W., Eisenberg A.: Phase separation behavior and crew-cut micelle formation of polystyrene- $b$-poly(acrylic acid) copolymers in solutions. Macromolecules, 30, 1001-1011 (1997).

[40] Cameron N. S., Corbierre M. K., Eisenberg A.: 1998 E.W.R. Steacie award lecture asymmetric amphiphilic block copolymers in solution: A morphological wonderland. Canadian Journal of Chemistry, 77, 13111326 (1999).

[41] Wang X. Y., Zhu J., Zhou D., Zhu X. L.: Reversible addition-fragmentation chain transfer free-radical polymerization of 2-(dimethylamino) ethyl methacrylate and synthesis of derived block polymer (in Chinese). Petrochemical Technology, 34, 536-540 (2005).
[42] Chen W-X., Fan X-D., Liu Y-Y.: Synthesis and characterization of amphiphilic triblock copolymers and their self-assembly in aqueous solution. Polymer Materials Science and Engineering, 22, 88-92 (2006).

[43] Kajiwara A., Matyjaszewski K.: Formation of block copolymers by transformation of cationic ring-opening polymerization to atom transfer radical polymerization (ATRP). Macromolecules, 31, 3489-3493 (1998).

[44] Lee S. B., Russell A. J., Matyjaszewski K.: ATRP synthesis of amphiphilic random, gradient, and block copolymers of 2-(dimethylamino)ethyl methacrylate and n-butyl methacrylate in aqueous media. Biomacromolecules, 4, 1386-1393 (2003).

[45] Matyjaszewski K., Göbelt B., Paik H-J., Horwitz C. P.: Tridentate nitrogen-based ligands in $\mathrm{Cu}$-based ATRP: A structure-activity study. Macromolecules, 34, 430-440 (2001).

[46] Mühlebach A., Gaynor S. G., Matyjaszewski K.: Synthesis of amphiphilic block copolymers by atom transfer radical polymerization (ATRP). Macromolecules, 31, 6046-6052 (1998).

[47] Neugebauer D., Zhang Y., Pakula T., Sheiko S. S., Matyjaszewski K.: Densely grafted and doublegrafted PEO brushes via ATRP. A route to soft elastomers. Macromolecules, 36, 6746-6755 (2003).

[48] Shinoda H., Miller P. J., Matyjaszewski K.: Improving the structural control of graft copolymers by combining ATRP with the macromonomer method. Macromolecules, 34, 3186-3194 (2001).

[49] Barim G., Demirelli K., Coskun M.: Conventional and atom transfer radical copolymerization of phenoxycarbonylmethyl methacrylate-styrene and thermal behavior of their copolymers. Express Polymer Letters, 1, 535-544 (2007).

[50] Lu X. J., Meng L. Z., Zhong X. X.: Novel fluorescent amphiphilic block copolymers: Photophysics behavior and interactions with DNA. Express Polymer Letters, 1, 356-363 (2007).

[51] Brandrup J., Immergut E. H.: Polymer handbook. Wiley, New York (1975).

[52] Cheng N., Hu S.: Solvent handbook. Chinese Chemical Engineering Press, Beijing (1986).

[53] Hickl P., Ballauff M., Jada A.: Small-angle X-ray contrast-variation study of micelles formed by poly (styrene)-poly(ethylene oxide) block copolymers in aqueous solution. Macromolecules, 29, 4006-4014 (1996).

[54] Zhang L., Yu K., Eisenberg A.: Ion-induced morphological changes in 'crew-cut' aggregates of amphiphilic block copolymers. Science, 272, $1777-$ 1779 (1996).

[55] Zhang L., Eisenberg A.: Multiple morphologies of 'crew-cut' aggregates of polystyrene-b-poly(acrylic acid) block copolymer. Science, 268, 1728-1731 (1995). 